\title{
Fitting \& Extrapolation of Turbine Performance Maps of Automotive Turbochargers
}

\author{
Adam Vondrák ${ }^{1,2, *}$ \\ ${ }^{1}$ Brno University of Technology, Institute of Automotive Engineering, Technická 2, 61669 Brno, \\ Czech Republic \\ ${ }^{2}$ Garrett Motion Czech Republic s.r.o., Tuřanka 100, 62700 Brno, Czech Republic
}

\begin{abstract}
The study presents a detailed analysis of a contemporary method for fitting and extrapolation of turbine performance maps of automotive turbochargers. The complete algorithm is implemented in a MATLAB ${ }^{\mathrm{TM}}$ based application with graphical user interface, the ultimate goal of which is to facilitate turbocharger performance data postprocessing. Guidelines are given on how to generate extrapolated maps from a fitted model considering the output data resolution. As a key validity criterion, fit quality of a sample measured turbine map is assessed using statistical and analytical methods. The report is concluded by a discussion of possible ways to improve the robustness of the algorithm with respect to input data of different origin and quality.
\end{abstract}

\section{Introduction}

The most appropriate turbocharger for a combustion engine is selected by the matching process. It must confirm that target engine power and torque can be achieved based on thermodynamic properties of applied turbine and compressor. These are described by performance maps, which include information about the interrelationship between rotating speed, pressure ratio, mass flow rate and efficiency.

Turbocharger performance maps can be acquired in several ways including (but not limited to) hot gas stand measurement and CFD based prediction. In each case, however, the product is a set of scattered data, which must be numerically fitted to enable inter/extrapolation to any feasible operating point by simulation software (see Figure 1).

State-of-the-art commercial products typically include working solutions for modelling of turbomachinery performance characteristics. GT-SUITE ${ }^{\mathrm{TM}}[1,2,3]$ can be mentioned as an example of the most widely used application for engine thermodynamics simulation and turbocharger matching (among other).

The aim of this work is to reproduce the industry-standard turbine map fitting method and analyse its properties, so it can be considered for a potential further use in post-processing of larger amounts of experimental data. MATLAB ${ }^{\mathrm{TM}}$ [4] environment has been selected for implementation of the algorithm for reasons of its availability and easy handling of input data files with help of built-in functions.

\footnotetext{
*Corresponding author: adam.vondrak@garrettmotion.com
} 


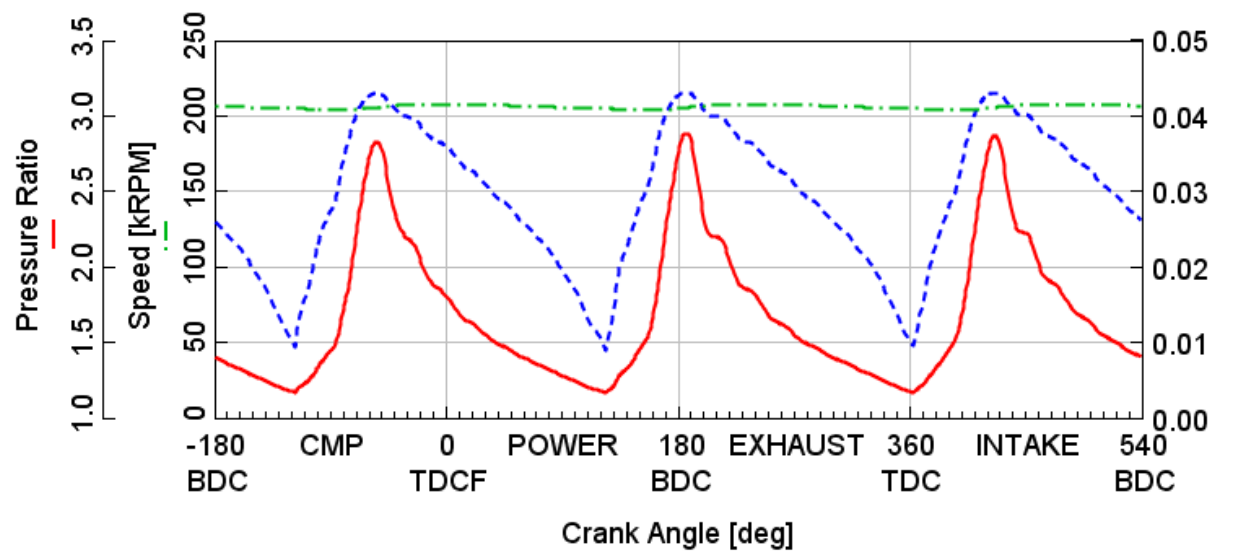

Fig. 1. An example of pressure ratio, corrected mass flow rate and turbine speed traces produced as part of three-cylinder engine thermodynamic cycle simulation in GT-SUITE (TDCF - top dead centre firing, TDC - top dead centre, BDC - bottom dead centre).

\section{Implementation of turbine map fitting method}

The common turbine performance model, as described in literature $[1,2]$, consists of five fitting functions for key thermodynamic properties of the machine. These are the optimum blade speed ratio $(B S R)$, maximum efficiency, optimum corrected mass flow rate, normalized efficiency and normalized mass flow rate. $B S R$ is defined as a ratio between the circumferential velocity of a turbine wheel and the isentropic spouting velocity, which would be obtained during an ideal expansion of the working gas between the entry and exit pressures of the stage. It can be calculated using the formula $[5,6]$

$$
B S R=\frac{u}{c_{0}}=\frac{\pi D \frac{n}{60}}{\sqrt{2 c_{p} T_{1} \text { tot }\left[1-\left(\frac{p_{2}}{p_{1} \text { tot }}\right)^{\frac{\kappa-1}{\kappa}}\right]}}
$$

where $u[\mathrm{~m} / \mathrm{s}]$ is circumferential velocity of the turbine wheel, $c_{0}[\mathrm{~m} / \mathrm{s}]$ is isentropic spouting velocity, $D[\mathrm{~m}]$ is wheel diameter, $n[\mathrm{rpm}]$ is frequency of rotation, $c_{\mathrm{p}}[\mathrm{J} / \mathrm{kg} / \mathrm{K}]$ is specific heat capacity at constant pressure, $T_{1_{-} \text {tot }}[\mathrm{K}]$ is inlet total temperature, $p_{I_{-} \text {tot }}[\mathrm{Pa}]$ is inlet total pressure, $p_{2}[\mathrm{~Pa}]$ is outlet static pressure and $\kappa[-]$ is ratio of specific heats.

\subsection{Pre-processing of turbine map}

A typical turbine map is obtained as a product of hot gas stand measurement $[5,7]$, where a complete turbocharger is mounted on the testing device and characterised under steady conditions. The result is a set of operating points defined by corrected or reduced rotating speed $(N T)$, pressure ratio $(P R T)$, corrected or reduced mass flow rate $(W T)$ and thermomechanical efficiency (EtaTM). Also, they are usually sorted into groups of constant rotating speed, which are referred to as speed lines.

Corrected (or reduced) quantities are used to make the performance map applicable to any operating conditions determined by inlet total pressure and temperature. The definition of corrected speed is $[5,6]$ 


$$
N T=n \sqrt{\frac{T_{r e f}}{T_{1 \_} t o t}}
$$

where $T_{\text {ref }}[\mathrm{K}]$ is reference temperature (usually $288 \mathrm{~K}$ ). Reduced speed would be obtained by omitting the reference temperature in Equation 2.

Corrected mass flow rate is defined as $[5,6]$

$$
W T=\dot{m} \frac{p_{\text {ref }}}{p_{1_{-} t o t}} \sqrt{\frac{T_{1_{-} t o t}}{T_{\text {ref }}}}
$$

where $\dot{m}[\mathrm{~kg} / \mathrm{s}]$ is mass flow rate, $p_{\text {ref }}[\mathrm{Pa}]$ is reference pressure (usually $101325 \mathrm{~Pa}$ ). Reduced mass flow rate would be obtained by dropping reference pressure and temperature in Equation 3.

Pressure ratio is determined by the inlet total pressure and outlet static pressure (according to a convention $[5,6])$ as follows

$$
P R T=\frac{p_{1 \_t o t}}{p_{2}}
$$

The first step of turbine map fitting process is to identify operating points of maximum efficiency within each speed line and save their indices (see Figure 2). Then, blade speed ratio is calculated at each operating point according to Equation 1.

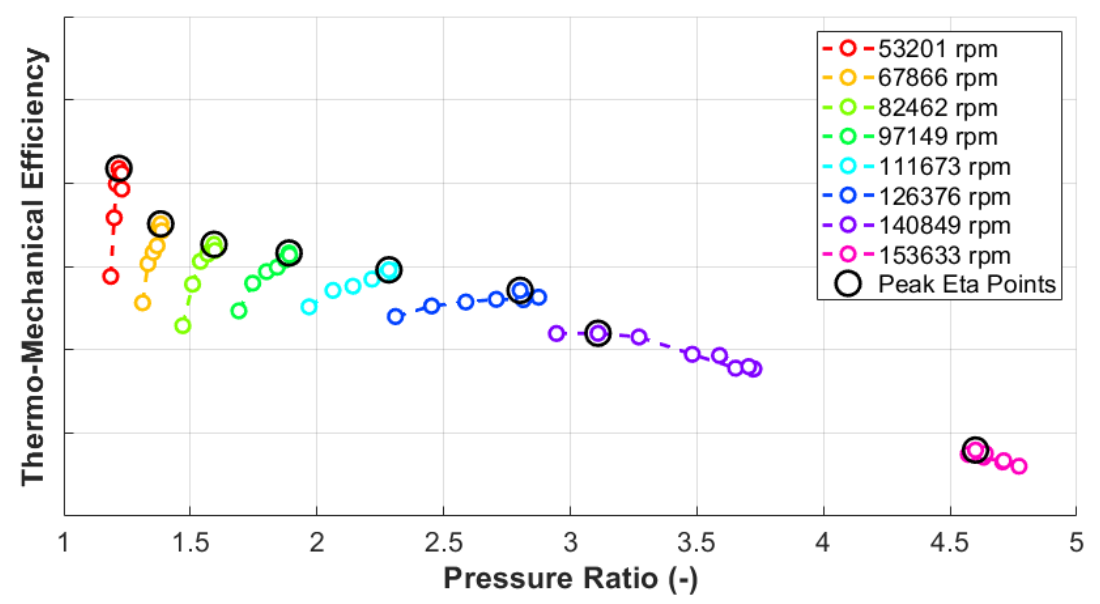

Fig. 2. Turbine efficiency map (measured on a gas stand) with highlighted operating points of maximum efficiency at each speed line (distinguished by colour).

\subsection{Fitting the optimum blade speed ratio}

Optimum blade speed ratio is linked to turbine operation with maximum efficiency at certain level of pressure ratio. This relationship is assumed to be linear [1,2], so the fitting function can be expressed as [10]

$$
B S R_{o p t}=k P R T+q
$$

where $B S R_{\text {opt }}[-]$ is the optimum blade speed ratio, $k$ [-] is the slope of the line and $q[-]$ is a constant offset. Parameters $k$ and $q$ can be determined using the least squares method $[8,10]$. 


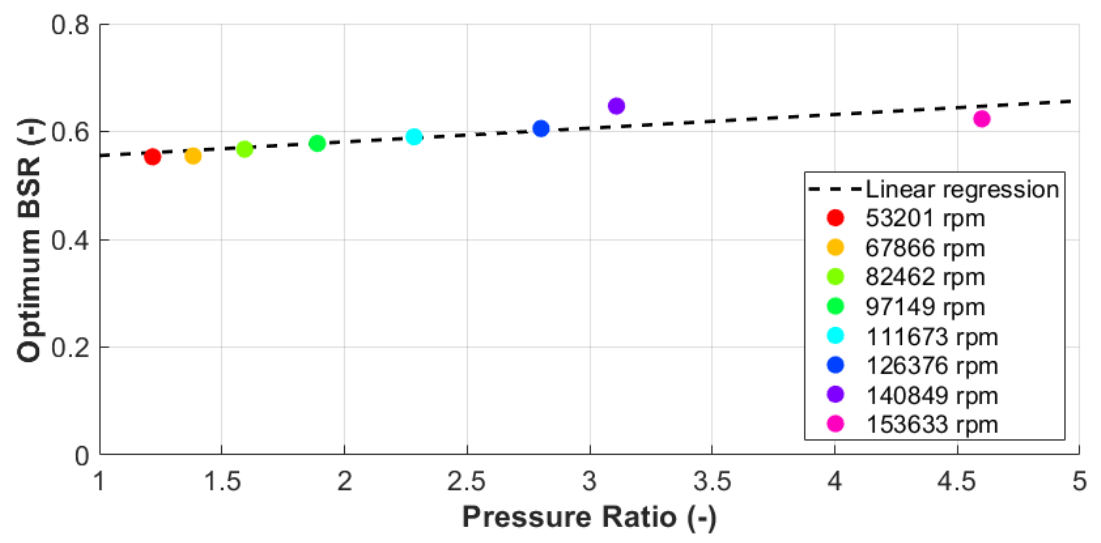

Fig. 3. Linear regression of optimum $B S R$ relative to $P R T$.

As soon as the fitting function for $B S R_{\text {opt }}$ is known, normalized blade speed ratio $\left(B S R_{\text {norm }}\right)$ can be calculated at each operating point of the turbine map. It is defined as $[1,2]$

$$
B S R_{\text {norm }}=\frac{B S R}{B S R_{\text {opt }}}
$$

where $B S R_{\text {opt }}$ is determined at the pressure ratio of the operating point being treated.

\subsection{Fitting the maximum efficiency}

Maximum efficiency points identified in the first step of the algorithm (see chapter 2.1) are fitted with respect to corrected speed $[1,2]$. The fitting function should be smooth, yet there is not enough reference in the literature that would suggest a specific one. Nevertheless, a spline curve can be recommended for its stability and easy definition of specific extrapolation requirements (the common approach is a constant value $[1,2]$ ).

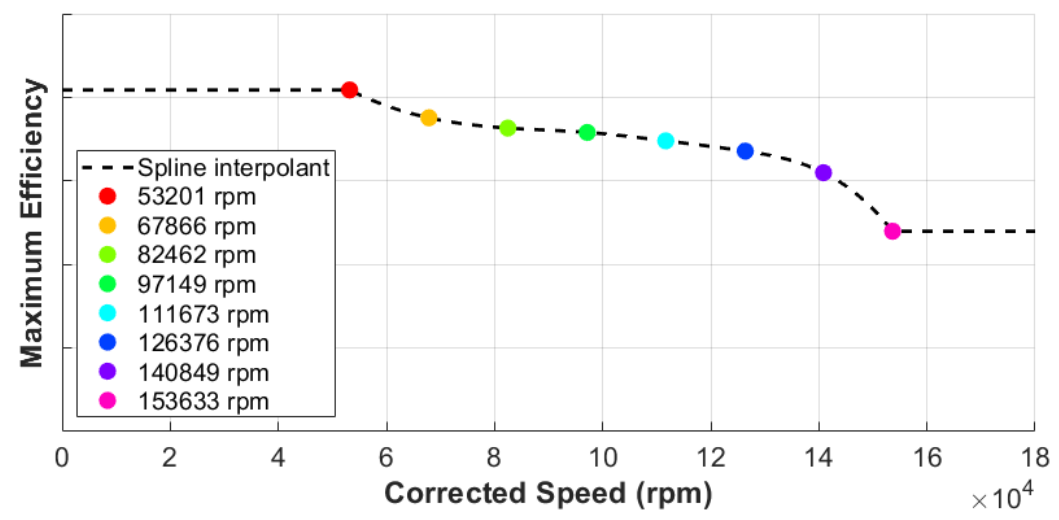

Fig. 4. Maximum efficiency spline with flat extrapolation.

With the fitting function for maximum efficiency available, normalized efficiency $\left(\right.$ EtaTM $\left._{\text {norm}}\right)$ can be calculated at each operating point of the map. The formula is $[1,2]$

$$
\operatorname{EtaTM}_{\text {norm }}=\frac{\text { EtaTM }_{\text {EtaTM }} \text { max }}{\text { Eta }}
$$


where $E t a T M_{\max }[-]$ is determined at the pressure ratio of the operating point being treated. Maximum efficiency function is, however, defined with respect to corrected speed, so the optimum speed for given pressure ratio must be calculated first. It can be done by merging the function for optimum $B S R$ (see Equation 5) and the definition of $B S R$ (see Equation 1). The resulting formula is

$$
n_{o p t}=(k P R T+q) \frac{\sqrt{2 c_{p} T_{1} t o t\left[1-\left(\frac{1}{P R T}\right)^{\frac{\kappa-1}{\kappa}}\right]}}{\frac{\pi D}{60}}
$$

where $n_{\mathrm{opt}}[\mathrm{rpm}]$ is the optimum rotating frequency at given PRT.

\subsection{Fitting the normalized efficiency}

According to the literature $[1,2]$, all turbine operating points should lie on a single curve when plotted in a diagram of normalized efficiency over normalized blade speed ratio (see Figure 5). In GT-SUITE, this curve is divided in two. For $B S R_{\text {norm }}<1$, the fitting function is exponentiation $[1,2]$

$$
\operatorname{EtaTM}_{\text {norm }}=1-\left(1-B S R_{\text {norm }}\right)^{b}
$$

where exponent $b[-]$ is a fitted parameter.

For $B S R_{\text {norm }}>1$, the fitting function is a parabola $[1,2]$

$$
\text { EtaTM }_{\text {norm }}=1-c\left(B S R_{\text {norm }}-1\right)^{2}
$$

where coefficient $c[-]$ is a fitted parameter that is also related to the intercept of the function with the x-axis. The value of $B S R_{\text {norm, }}$ at which the $\mathrm{x}$-axis is crossed, can be determined using an equation

$$
B S R_{\text {int }}=\frac{1}{c^{2}}+1
$$

where $B S R_{\text {int }}[-]$ is the value of normalized $B S R(>1)$ at zero normalized efficiency.

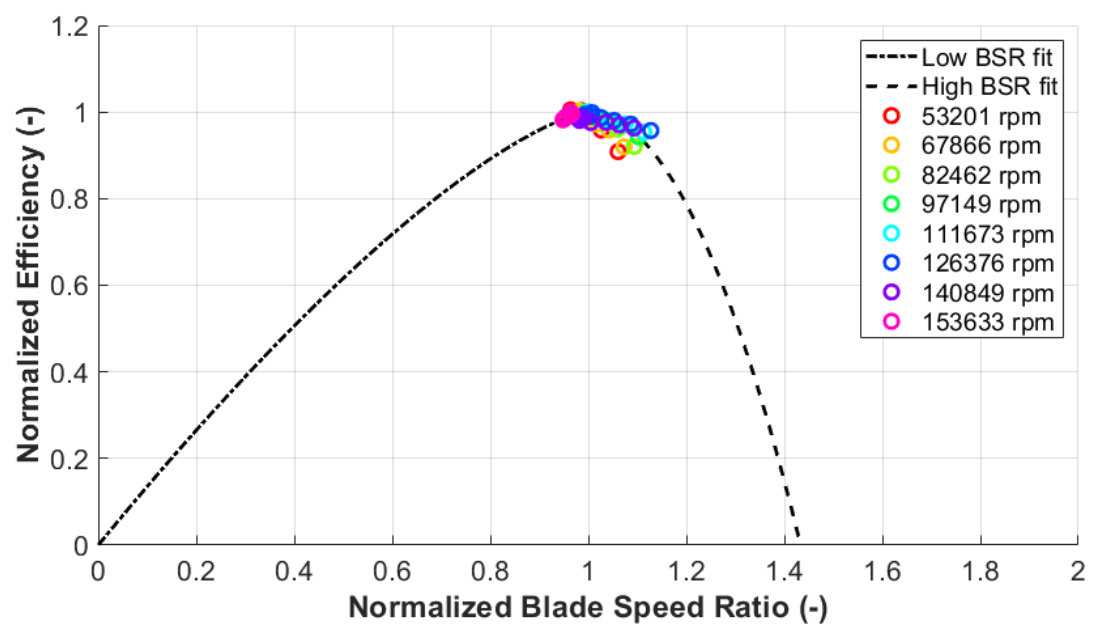

Fig. 5. Normalized efficiency fitted with respect to normalized blade speed ratio. 


\subsection{Fitting the optimum corrected mass flow rate}

A value of optimum corrected mass flow rate is associated to each operating point of maximum efficiency at each speed line (see Chapter 2.1). These points are fitted with respect to corrected speed and the function is supposed to pass through the origin of the coordinate system $[1,2]$. A spline curve is a convenient interpolant again (see Chapter 2.3).

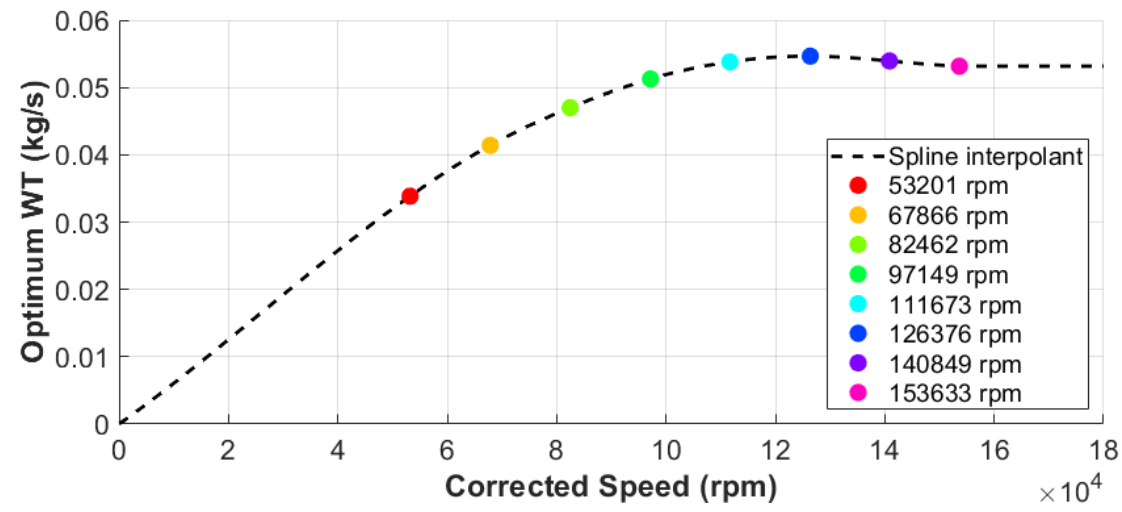

Fig. 6. Optimum corrected mass flow rate fitted by a spline passing through the origin of the coordinate system with flat extrapolation to high corrected speeds.

With the fitting function for optimum corrected mass flow rate available, normalized mass flow rate $\left(W T_{\text {norm }}\right)$ can be calculated at each operating point according to $[1,2]$

$$
W T_{\text {norm }}=\frac{W T}{W T_{o p t}}
$$

where $W T_{\mathrm{opt}}[\mathrm{kg} / \mathrm{s}]$ is optimum corrected mass flow rate at the pressure ratio of the operating point being treated. The optimum mass flow rate function is, however, defined with respect to corrected speed, so the optimum speed for given pressure ratio must be determined using Equation 8 as described in Chapter 2.3.

\subsection{Fitting the normalized mass flow rate}

According to the literature $[1,2]$, all turbine operating points should lie on a single curve when plotted as normalized mass flow rate over normalized blade speed ratio (see Figure 7).

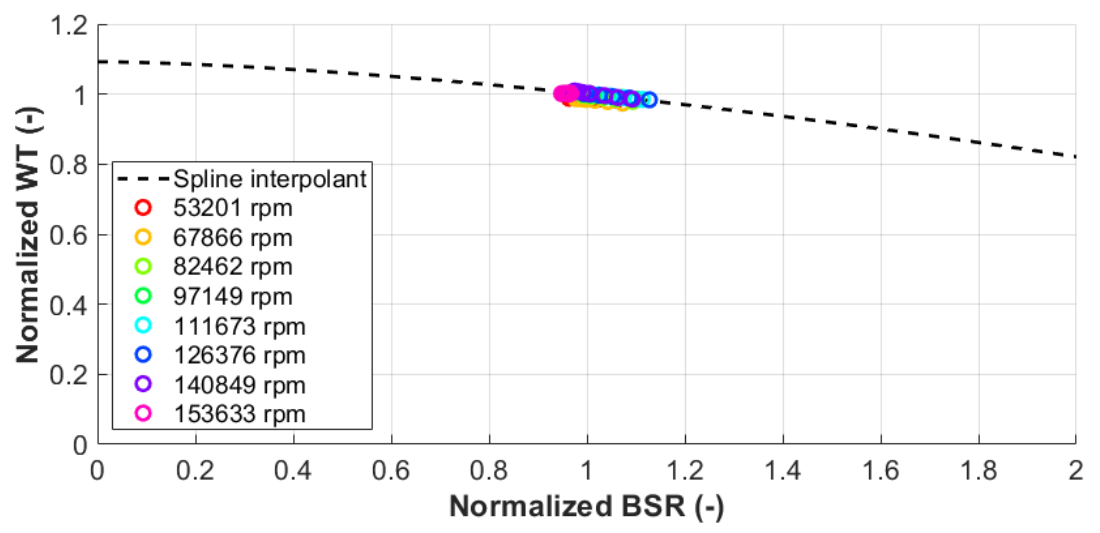

Fig. 7. Normalized mass flow rate fitted with respect to normalized blade speed ratio. 
In GT-SUITE, normalized mass flow rate is fitted by exponentiation in the form $[1,2]$

$$
W T_{\text {norm }}=c_{m}+B S R_{\text {norm }}^{m}\left(1-c_{m}\right)
$$

where constant $c_{\mathrm{m}}[-]$ and exponent $m[-]$ are fitted parameters.

\section{Back-calculation of turbine performance charts}

Once turbine performance model is fitted to the source data, basic properties of the stage can be visualized in form of fully extrapolated efficiency and mass flow rate maps.

\subsection{Extrapolated efficiency map}

A turbine efficiency map consists of speed lines, which are made of operating points of constant corrected speed. It is convenient to select the same speeds that were identified in the source data, so its agreement with the fit is easily observable.

The value of turbine efficiency $(E t a T M)$ is obtained as a product of normalized efficiency $\left(\right.$ EtaTM $\left._{\text {norm }}\right)$ and maximum efficiency $\left(\right.$ EtaTM $\left._{\max }\right)$ for certain pressure ratio (see Equation 7). Fitting function for $E t a T M_{\max }$ is, however, defined with respect to optimum corrected speed, so it must be calculated using Equation 8 first. $E t a T M_{\text {norm }}$, on the other hand, is fitted relative to normalized blade speed ratio $\left(B S R_{\text {norm }}\right)$ according to Equations 9 and 10 . Finally, $B S R_{\text {norm }}$ is defined by Equation 6 and depends on $B S R$ (see Equation 1) and $B S R_{\text {opt }}$ (see Equation 5). Pressure ratio $(P R T)$, therefore, remains the only independent variable and enters the process as a generated set of equidistant values.

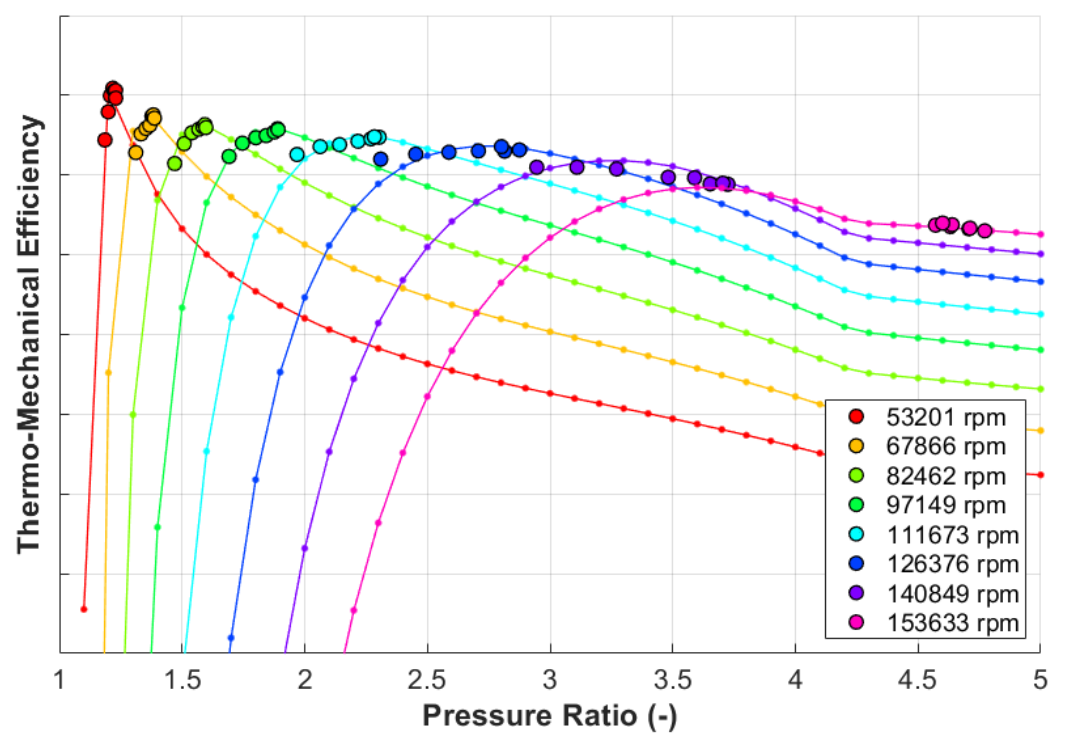

Fig. 8. Extrapolated efficiency map with $P R T$ points defined as an equally spaced set.

The curves of low speed lines in Figure 8 have notably poorer resolution than the highspeed ones, which is best visible near the peak efficiency points. One way to improve it would be by increasing the number of points constituting each speed line, however it may be inconvenient for sharing or using it as an input in engine thermodynamics simulation. Therefore, the input $P R T$ coordinates can be derived from a set of equally spaced $B S R$ values (see Equation 1) using a formula 


$$
P R T=\left[1-\left(B S R^{-1} \frac{\pi D n}{\sqrt{2 c_{p} T_{1} t o t}}\right)^{2}\right]^{\frac{\kappa}{1-\kappa}}
$$

In Figure 9, the number of points per speed line is the same or lower than in Figure 8.

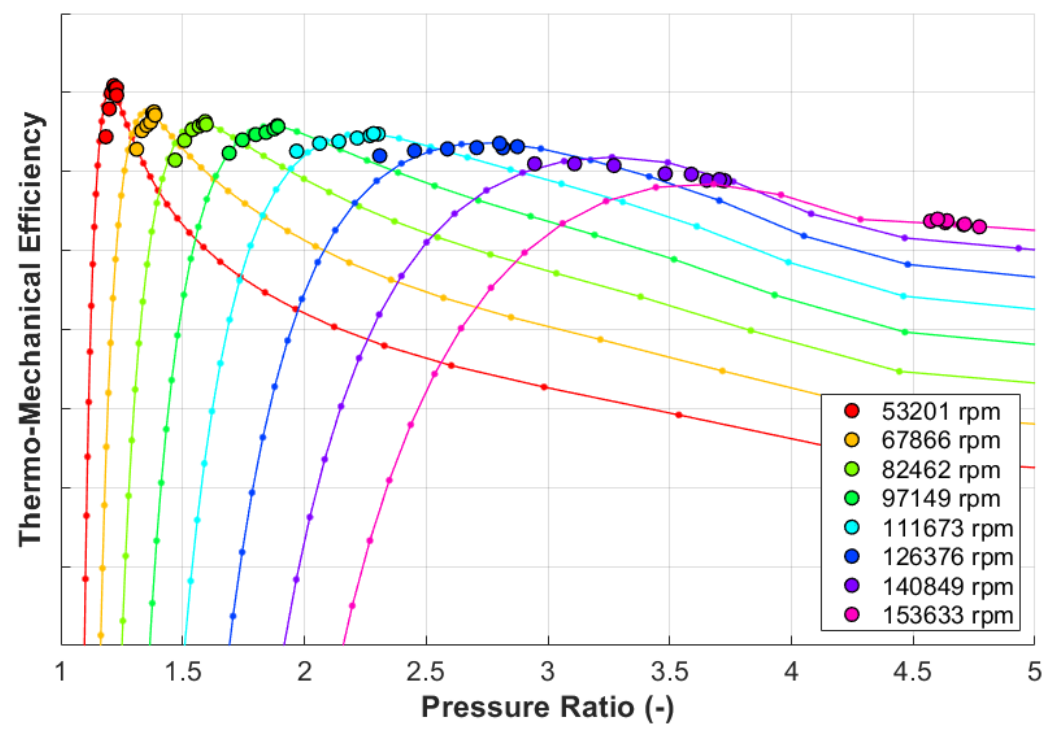

Fig. 9. Extrapolated efficiency map with $P R T$ points derived from an equally spaced set of $B S R$.

\subsection{Extrapolated corrected mass flow rate map}

A turbine corrected mass flow rate map consists of speed lines plotted over the pressure ratio (see Figure 10). The process of generating the data is analogical to the case of efficiency (see Chapter 3.1), only with a different dependent variable.

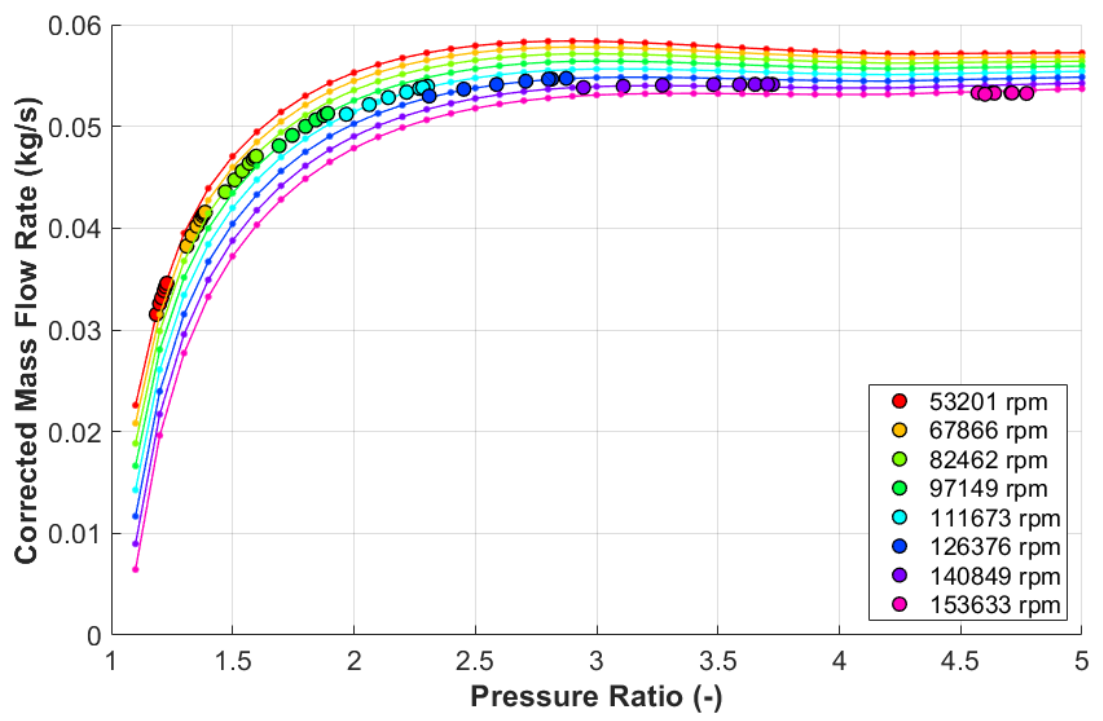

Fig. 10. Extrapolated mass flow map with $P R T$ points defined as an equally spaced set. 
The value of corrected mass flow rate $(W T)$ is obtained as a product of normalized mass flow rate $\left(W T_{\text {norm }}\right)$ and optimum mass flow rate $\left(W T_{\text {opt }}\right)$ for certain pressure ratio (see Equation 12). Fitting function for $W T_{\text {opt }}$ is, however, defined with respect to optimum corrected speed, so it must be calculated using Equation 8 first. $W T_{\text {norm, }}$ on the other hand, is fitted relative to normalized blade speed ratio $\left(B S R_{\text {norm }}\right)$ according to Equations 9 and 10 . Finally, $B S R_{\text {norm }}$ is defined by Equation 6 and depends on $B S R$ (see Equation 1) and $B S R_{\text {opt }}$ (see Equation 5). Pressure ratio $(P R T)$, therefore, remains the only independent variable and enters the process as a generated set of equidistant values.

\section{Fit quality assessment}

The quality of the model can be evaluated in terms of difference between the source data and fit values obtained for the same set of independent variables (corrected speed and pressure ratio). The common way to do that is by means of statistical methods [8,9], however it is worth to perform a visual analysis of characteristic parts of each extrapolated map too.

\subsection{Statistical approach}

In data fitting, the initial step of any statistical analysis is to determine the error between each source data point and the model. There are two turbine performance parameters that play the major role: efficiency and mass flow rate. The error values must be determined independently for each. To the basic statistics belong the minimum, maximum, mean, median, mean squared error (MSE) and root-mean-square error (RMSE) $[8,9,10]$.

Table 1. Statistics of sample turbine map fit quality

\begin{tabular}{|c|c|c|}
\hline & Efficiency & $\begin{array}{c}\text { Normalized corrected } \\
\text { mass flow rate }\end{array}$ \\
\hline Minimum error & $-5.09 \%$ & $-1.28 \%$ \\
\hline Maximum error & $2.85 \%$ & $0.5 \%$ \\
\hline Mean error & $-0.42 \%$ & $-0.28 \%$ \\
\hline Median error & $-0.27 \%$ & $-0.07 \%$ \\
\hline Mean absolute error & $0.77 \%$ & $0.43 \%$ \\
\hline MSE & 1.48 & 0.34 \\
\hline RMSE & $1.22 \%$ & $0.58 \%$ \\
\hline
\end{tabular}

The fit is not perfectly neutral as both mean and median error are different from zero for both efficiency and corrected mass flow rate (see Table 1). Nevertheless, the difference is lower than half a percentage point, which is one tenth of the biggest efficiency deviation. The distribution of error sizes can also be visualised by means of a histogram $[8,9]$. Figure 11 shows that most of the efficiency error values fall in the interval from $-1 \%$ to $1 \%$, which corresponds to approximately three quarters of the total number of data points. Relative corrected mass flow rate error is even less scattered with zero occurrence below $-2 \%$ and above $1 \%$. 

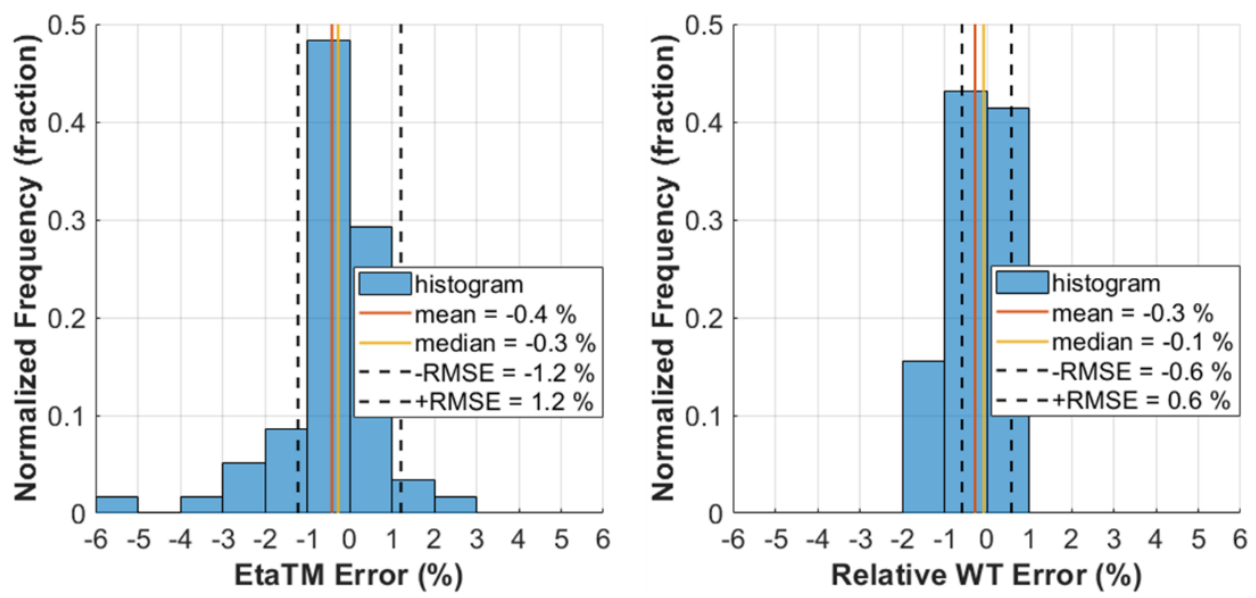

Fig. 11. Histograms of error size distribution for efficiency (left) and mass flow rate (right) fits.

A few data points, on the other hand, fall in the bins of efficiency error bigger than $3 \%$, which makes it worth to visualize what speed line they belong to. This can be done by plotting the error values relative to the index of each operating point (see Figure 12).
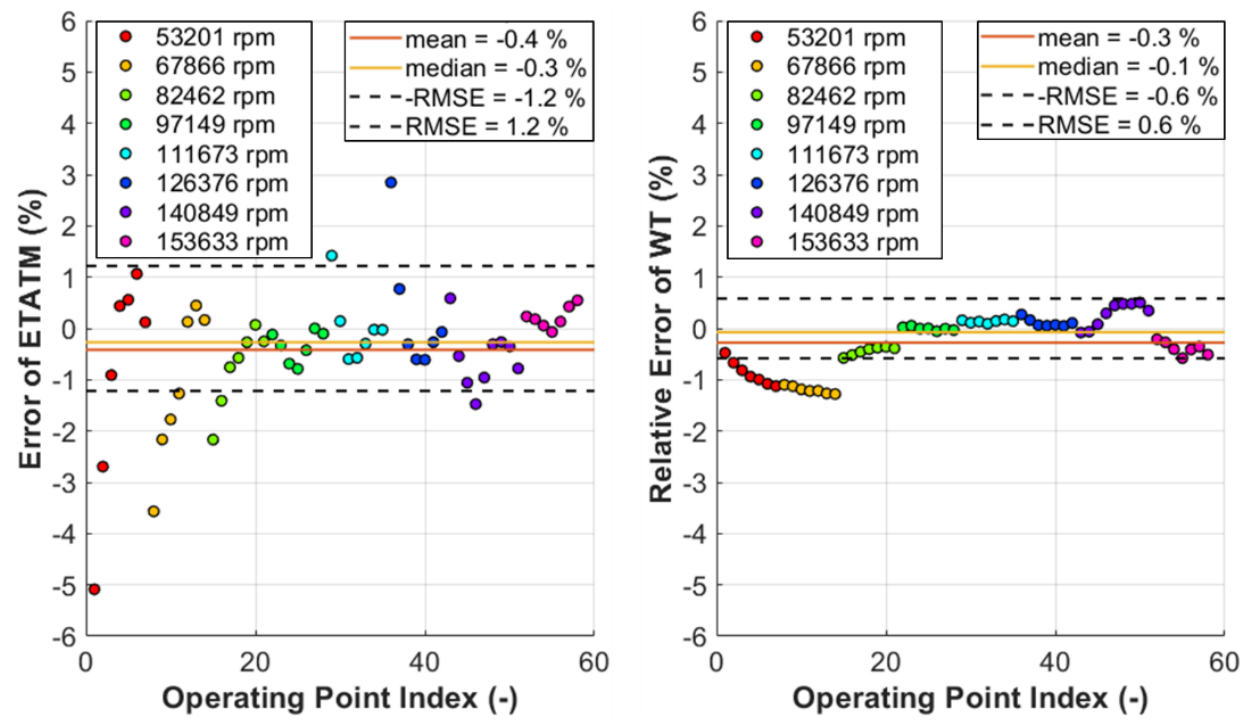

Fig. 12. Error size vs. data point index for efficiency (left) and mass flow rate (right) fits.

From the assessment of Figure 12 follows that the biggest error values are mostly connected with the lowest speed lines. This is partly due to the shape of low speed lines, which is sharper in an efficiency chart (see Figure 9) and steeper in a corrected mass flow rate chart (see Figure 10), so the values of dependent variables are sensitive to PRT. Also, mass flow rate can be measured directly on a gas stand, which makes the data less noisy.

\subsection{Analytical approach}

Apart from the statistical evaluation, it is worth to visually check the shape of extrapolated turbine performance maps too. One way to do that is by examining the maximum efficiency curve plotted over the pressure ratio in an extrapolated efficiency map (see Figure 13). 


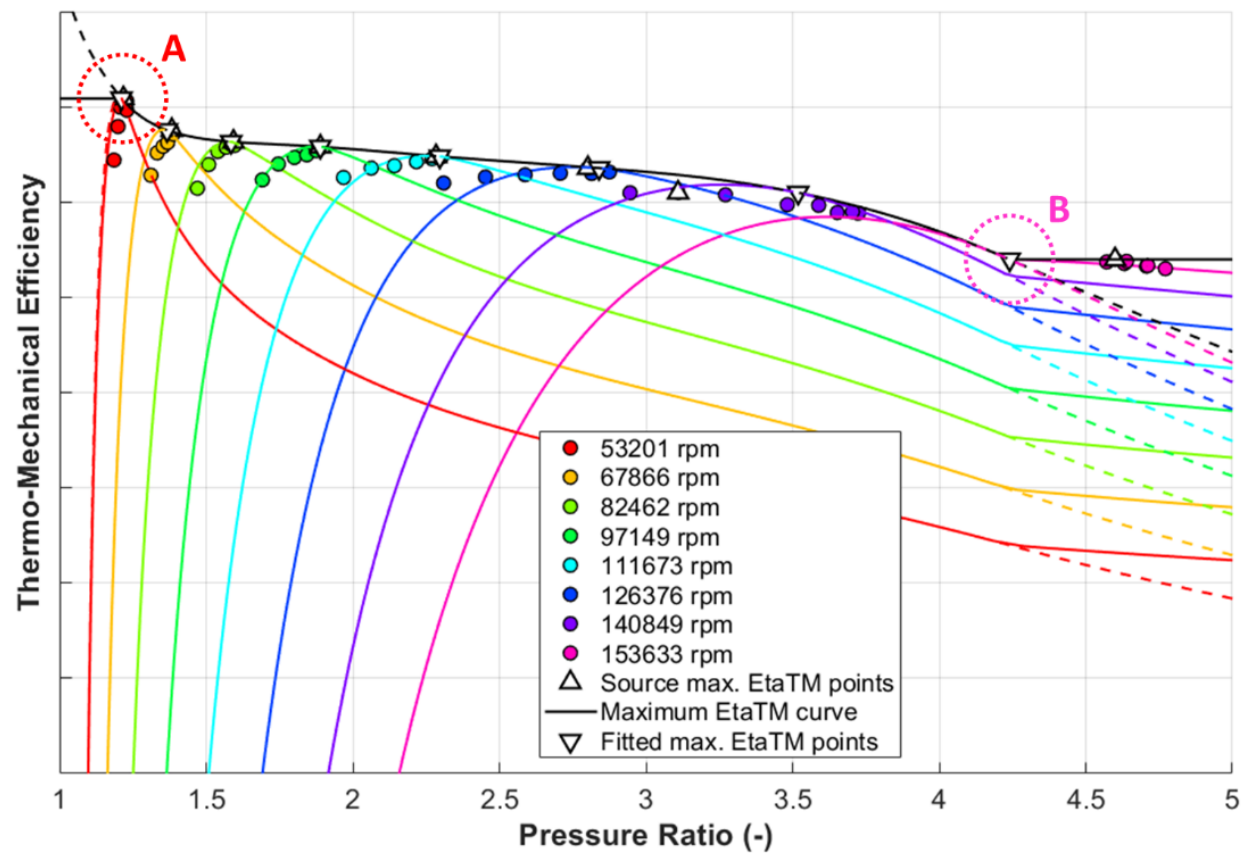

Fig. 13. Extrapolated efficiency map with maximum efficiency curve and points. $A$ and $B$ mark endpoints of main spline part of maximum efficiency curve (tangential extrapolation dashed).

In Figure 13, discrepancy can be seen between the location of maximum efficiency points derived from the model and those of the source map. The reason for the shift of their PRT coordinates is that optimum $B S R$ function is defined as a linear regression (in the least squares sense $[8,10])$ of source-map operating points of maximum efficiency, so the fit does not necessarily pass through all of them (see Figure 3 ). The PRT coordinate of each maximum efficiency point must be determined iteratively using Equation 14, because optimum $B S R$, as one of its inputs, depends on PRT too (see Equation 5).

Further, areas $A$ and $B$ in Figure 13 mark the points, where maximum efficiency curve transitions from the main spline part defined by the source map data to extrapolation regions (see Figure 4). One benefit of the flat extrapolation is that outlying source map points are still reasonably represented by the turbine performance model (see operating points to the right of area $B$ in Figure 13). This is possible, since endpoint efficiencies of the main spline part were defined using the peak-efficiency points of the lowest and the highest speed line of the source map, so the extrapolated curve must pass through them.

On the other hand, two sharp break points can be identified on the max. efficiency curve in Figure 13 (marked by areas $A$ and $B$ ), which is unphysical. One way to cope with that would be a tangential linear extrapolation instead of the flat one. The issue is, however, that the maximum efficiency curve may no longer pass through the operating points of maximum efficiency at the highest and the lowest speed line, which impacts the agreement between the fitted model and the source data (see dashed lines in Figure 13). Furthermore, extrapolated efficiency may, in some cases, rise above one or fall below zero (where it is not desired).

In case of corrected mass flow rate, the two areas of extrapolation can be identified in the corresponding map too (see Figure 14). The optimum corrected mass flow curve is, however, defined for corrected speeds starting at zero (corresponds to $P R T=1$ ), so the area $A$ does not mark any break point. Although the extrapolation to high corrected speeds is still flat, the slope of optimum corrected mass flow rate is almost zero in this region, so the transition is smooth again in the area $B$ in Figure 14 . 


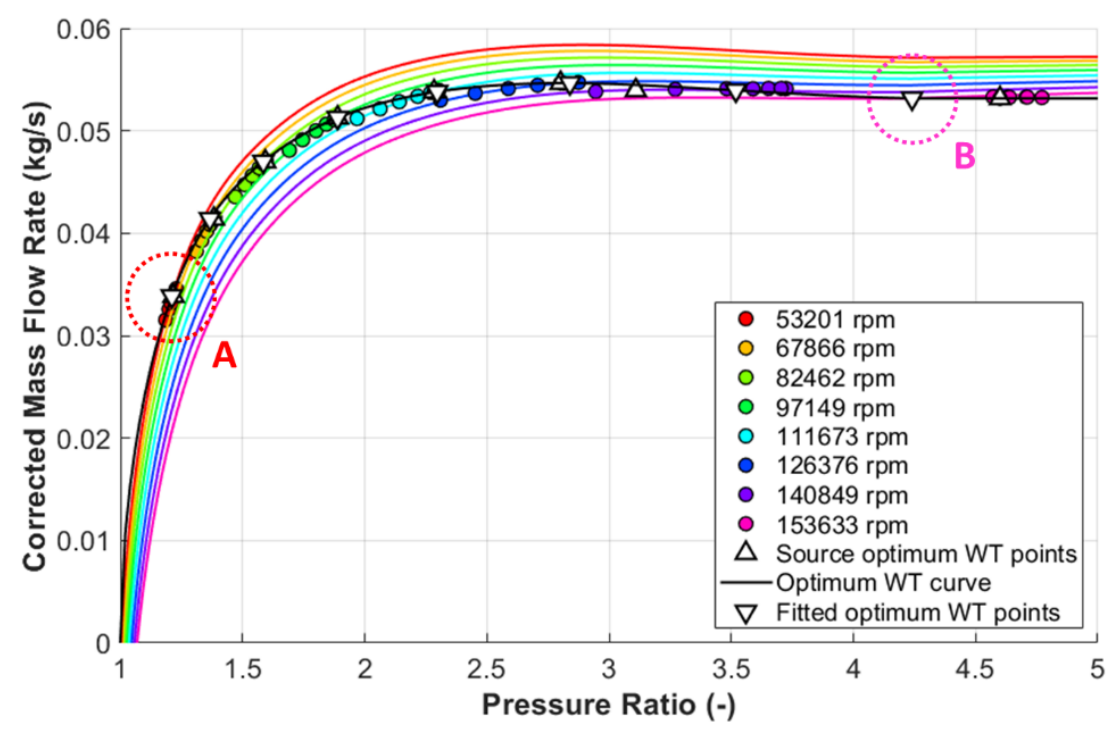

Fig. 14. Extrapolated mass flow map with optimum corrected mass flow rate curve and points. $A$ and $B$ mark endpoints of the main spline part of optimum corrected mass flow rate curve.

\section{Robustness check}

The above described methodology proved to be reasonable for modelling of the sample turbine stage performance. Now, it should be checked that the same process can be repeated for a different data set too. Another common way to obtain turbine performance map is by means of dynamometer testing. A turbine stage alone (without a compressor) is mounted on the stand, while the power output is measured directly on the turbine shaft. A sample fitted turbine dyno map is shown in Figure 15.

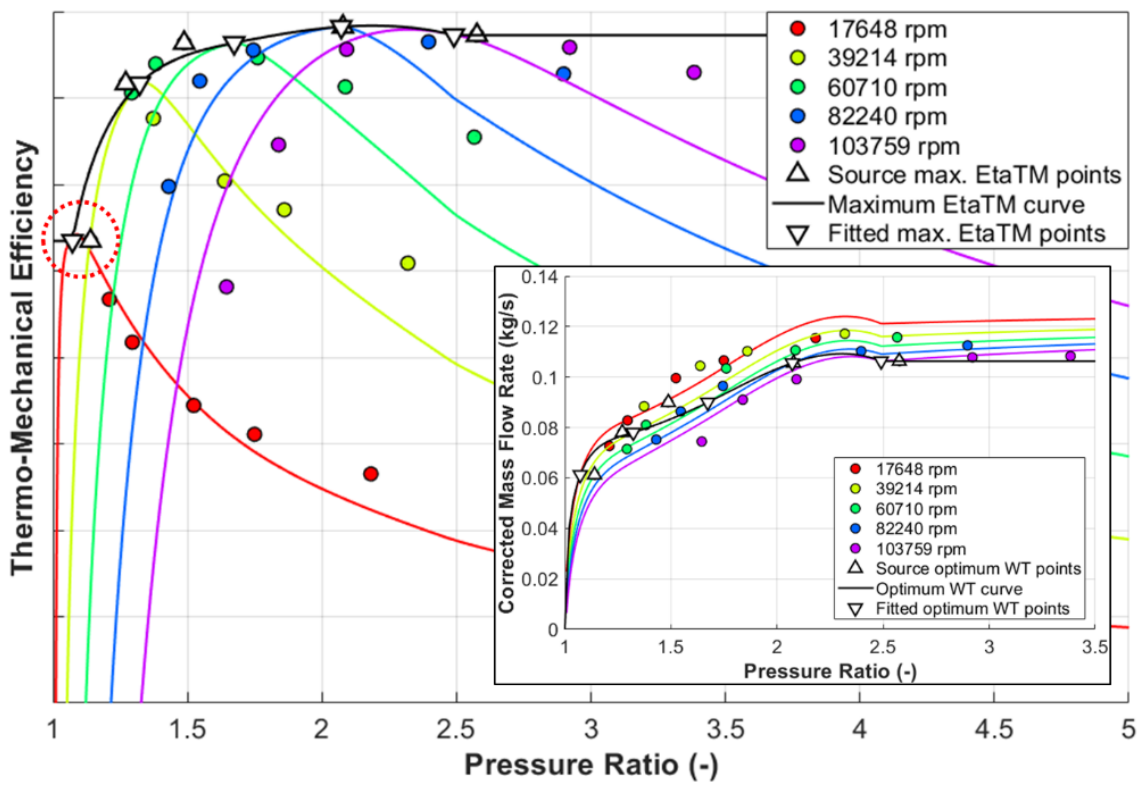

Fig. 15. Extrapolated efficiency and mass flow maps for turbine data acquired using a dynamometer. 
The fitting method failed in determining the maximum efficiency at low corrected speeds, where the measured data include sub-optimal operating points only (see Figure 15). Therefore, the resulting extrapolated efficiency map includes areas of significant distortion, which makes it useless for engine thermodynamics simulation or other modes of use.

\section{Conclusion}

It can be concluded that the fitted model represents the sample gas stand turbine map relatively well, however evidence can be found that it is not always the case. Especially in situations, when source operating points lie far away from the maximum efficiency area, the resulting fit is very poor (see dyno map in Figure 15).

One way to reduce model distortion would be to manually remove outlying points, which can generally help, when source data includes erroneous values. With the dyno map, however, complete speed lines would have to be deleted in order to prevent maximum efficiency misinterpretation. Not only can it lead to unwanted data loss, but there is no guarantee the remaining speed lines include true maximum efficiency points too.

As an alternative approach, optimization methods could be employed to search for the best properties of fitting functions that would yield the lowest overall fit error. This idea, however, exceeds the scope of this paper.

This work could be completed thanks to turbocharger performance data provided by Garrett Motion Inc. Also, consultations of prof. Ing. Josef Štětina, Ph.D., director of Institute of Automotive Engineering at Faculty of Mechanical Engineering of Brno University of Technology, are gratefully acknowledged.

\section{References}

1. A. Pesyridis, W. S-I. W. Salim, R. F. Martinez-Botas, $10^{\text {th }}$ International Conference on Turbochargers and Turbocharging, ISBN:9780857096135, p. 203-218 (Woodhead Publishing Ltd., Cambridge, 2012)

2. Gamma Technologies, GT-SUITE Flow Theory Manual, p. 99-102 (2019)

3. Gamma Technologies, GT-SUITE, https://www.gtisoft.com/, (2020)

4. MathWorks, MATLAB, https://www.mathworks.com/, (2020)

5. N. Baines, Fundamentals of Turbocharging, ISBN:0933283148, 1 (Concepts NREC, Vermont, 2005)

6. N. Watson, M. S. Janota, Turbocharging the internal combustion engine, ISBN:0333242904, 1 (The Macmillan Press Ltd., London, 1982)

7. Kratzer Automation, Test benches for turbochargers, https:/www.kratzerautomation.com/testsystems/en/test-bench-solutions/turbocharger/, (2020)

8. V. N. Tutubalin, Teorie pravděpodobnosti, 1 (SNTL, Prague, 1978)

9. L. Žák, Popisná statistika, https://mathonline.fme.vutbr.cz/download.aspx?id_file=475, (2006)

10. Z. Karpíšek, Reg. analýza, https://mathonline.fme.vutbr.cz/download.aspx?id_file=524, (2006) 